\title{
Nonlinear Jordan Triple Derivations of Triangular Algebras
}

\section{Hongxia Li}

School of Science, Southwest University of Science and Technology, Mianyang, China Email: 474072723@qq.com

Received 10 October 2014; revised 10 November 2014; accepted 8 December 2014

Copyright (C) 2014 by author and Scientific Research Publishing Inc.

This work is licensed under the Creative Commons Attribution International License (CC BY). http://creativecommons.org/licenses/by/4.0/

(c) (i) Open Access

\section{Abstract \\ In this paper, it is proved that every nonlinear Jordan triple derivation on triangular algebra is an additive derivation.}

\section{Keywords}

\section{Nonlinear Jordan Triple Derivations, Triangular Algebras, Derivation}

\section{Introduction}

Let $\mathcal{R}$ be a commutative ring with identity and $\mathcal{A}$ be an $\mathcal{R}$-algebra. A linear map $\delta: \mathcal{A} \rightarrow \mathcal{A}$ is called a derivation if $\delta(A B)=\delta(A) B+A \delta(B)$ for all $A, B \in \mathcal{A}$. Additive (linear) derivations are very important maps both in theory and applications, and were studied intensively. More generally, we say that $\delta$ is a Jordan triple derivation if $\delta\left(\frac{1}{2}(A B C+C B A)\right)=\frac{1}{2}[\delta(A) B C+A \delta(B) C+A B \delta(C)+\delta(C) B A+C \delta(B) A+C B \delta(A)]$

for all $A, B, C \in \mathcal{A}$. If the linearity in the definition is not required, the corresponding map is said to be a nonlinear Jordan triple derivation. It should be remarked that there are several definitions of linear Jordan derivations and all of them are equivalent as long as the algebra $\mathcal{A}$ is 2-torsion free. We refer the reader to [1] for more details and related topics. But one can ask whether the equivalence is also true on the condition of nonlinear, and we are still unable to answer this question.

The structures of derivations, Jordan derivations and Jordan triple derivations were systematically studied. Herstein [2] proved that any Jordan derivation from a 2-torsion free prime ring into itself is a derivation, and the famous result of Brešar ([1], Theorem 4.3) states that every Jordan triple derivation from a 2-torsion free semiprime ring into itself is a derivation. For other results, see [3]-[9] and the references therein.

Let $\mathcal{A}$ and $\mathcal{B}$ be two unital algebras over a commutative ring $\mathcal{R}$, and let $\mathcal{M}$ be a unital $(\mathcal{A}, \mathcal{B})$-bi- 
module, which is faithful as a left $\mathcal{A}$-bimodule, that is, for $A \in \mathcal{A}, A \mathcal{M}=0 \Rightarrow A=0$ and a right $\mathcal{B}$-bimodule, that is, for $B \in \mathcal{A}, \mathcal{M} B=0 \Rightarrow B=0$. Recall the algebra $\mathcal{T}=\operatorname{Tri}(\mathcal{A}, \mathcal{M}, \mathcal{B})=\left\{\left(\begin{array}{ll}a & m \\ 0 & b\end{array}\right): a \in \mathcal{A}, b \in \mathcal{B}, m \in \mathcal{M}\right\}$ under the usual matrix addition and formal matrix multiplication is called a triangular algebra [10]. Recently, Zhang [11] characterized that any Jordan derivation on a triangular algebra is a derivation. In this paper we present result corresponding to [11] (Theorem 2.1) for non-linear Jordan triple derivations (there is no linear or additive assumption) on an important algebra: triangular algebra.

As a notational convenience, we will adopt the traditional representations. Let us write $P=\left(\begin{array}{cc}I_{\mathcal{A}} & 0 \\ 0 & 0\end{array}\right)$, $Q=\left(\begin{array}{cc}0 & 0 \\ 0 & I_{\mathcal{B}}\end{array}\right)$ and $I=\left(\begin{array}{cc}I_{\mathcal{A}} & 0 \\ 0 & I_{\mathcal{B}}\end{array}\right)$ for the identity matrix of the triangular algebra $\mathcal{T}$.

\section{The Main Results}

In this note, our main result is the following theorem.

Theorem 2.1. Let $\mathcal{A}$ and $\mathcal{B}$ be unital algebras over a 2-torsion free commutative ring $\mathcal{R}$, and $\mathcal{M}$ be a unital $(\mathcal{A}, \mathcal{B})$-bimodule, which is faithful as a left $\mathcal{A}$-bimodule and a right $\mathcal{B}$-bimodule. Let

$\mathcal{T}=\operatorname{Tri}(\mathcal{A}, \mathcal{M}, \mathcal{B})$ be the triangular algebra; if $\delta$ is a nonlinear Jordan triple derivation on $\mathcal{T}, \delta$ is an additive derivation.

Lemma 2.1. If $\delta$ is a nonlinear Jordan triple derivation on an upper triangular algebra $\mathcal{T}$ generated by $P=P^{2} \in \mathcal{T}, \quad \delta(P)=P T-T P$ with $T=\delta(P) \in \mathcal{T}$.

Proof. It follows from the fact $P=P^{2}$ that $\delta(P)=\delta(P) P+P \delta(P) P+P \delta(P)$, which implies that $P \delta(P) P=Q \delta(P) Q=0$. Thus we have from the fact that $Q \delta(P) P=0$ that $\delta(P)=P \delta(P) Q=P T-T P$, where $T=\delta(P) \in \mathcal{T}$.

Now define $d(A)=\delta(A)-(A T-T A)$ for each $A \in \mathcal{T}$. Clearly, $d$ is also a nonlinear Jordan triple derivation from $\mathcal{T}$ into itself. It follows from Lemma 2.1 that $d(P)=d(Q)=0$.

Lemma 2.2. $d(0)=0$.

Proof. Clearly, $d(0)=d(0) 00+0 d(0) 0+00 d(0)=0$.

Lemma 2.3. $d\left(\mathcal{T}_{i j}\right) \subseteq \mathcal{T}_{i j}, 1 \leq i \leq j \leq 2$.

Proof. Firstly, we prove that $d(2 P) \in \mathcal{T}_{11}$. It is clear that $d(2 P)=d\left(\frac{1}{2}(P \cdot 2 P \cdot P+P \cdot 2 P \cdot P)\right)=P d(2 P) P$, which implies that $d(2 P) \in \mathcal{T}_{11}$.

Let $A_{12} \in \mathcal{T}_{12}, \quad d\left(A_{12}\right)=d\left(\frac{1}{2}\left(2 P \cdot A_{12} \cdot Q+Q \cdot A_{12} \cdot 2 P\right)\right)=\frac{1}{2}\left[d(2 P) A_{12}+2 P d\left(A_{12}\right) Q\right]$. Since $d(2 P) A_{12} \in \mathcal{T}_{12}$, we get $d\left(A_{12}\right) \in \mathcal{T}_{12}$.

Let $A_{11} \in \mathcal{T}_{11}, \quad d\left(A_{11}\right)=d\left(\frac{1}{2}\left(P \cdot A_{11} \cdot P+P \cdot A_{11} \cdot P\right)\right)=P d\left(A_{11}\right) P$, and thus $d\left(A_{11}\right) \in \mathcal{T}_{11}$.

Similarly, one can check that $d\left(\mathcal{T}_{22}\right) \subseteq \mathcal{T}_{22}$.

Lemma 2.4. $d(2 P)=d(2 Q)=0$.

Proof. For any $A_{12} \in \mathcal{T}_{12}$, it follows from Lemma 2.3 that

$d\left(A_{12}\right)=d\left(\frac{1}{2}\left(2 P \cdot P \cdot A_{12}+A_{12} \cdot P \cdot 2 P\right)\right)=\frac{1}{2}\left[d(2 P) A_{12}+2 P d\left(A_{12}\right)\right]$. This implies that $P d(2 P) A_{12}=0$. Since $P \mathcal{T Q}$ is a faithful left $P \mathcal{T P}$-module, we have that

$$
P d(2 P) P=0 .
$$

It follows from $d(2 P) \in \mathcal{T}_{11}$, we have $d(2 P)=0$. Similarly, we can get that $d(2 Q)=0$.

Lemma 2.5. For any $A_{11}, B_{11} \in \mathcal{T}_{11}, A_{12}, B_{12} \in \mathcal{T}_{12}, A_{22}, B_{22} \in \mathcal{T}_{22}$, we have

(1) $d\left(A_{11} B_{12}\right)=d\left(A_{11}\right) B_{12}+A_{11} d\left(B_{12}\right)$, (2) $d\left(A_{12} B_{22}\right)=d\left(A_{12}\right) B_{22}+A_{12} d\left(B_{22}\right)$,

(3) $d\left(A_{11} B_{11}\right)=d\left(A_{11}\right) B_{11}+A_{11} d\left(B_{11}\right)$, (4) $d\left(A_{22} B_{22}\right)=d\left(A_{22}\right) B_{22}+A_{22} d\left(B_{22}\right)$. 
Proof. (1) For any $A_{11} \in \mathcal{T}_{11}, B_{12} \in \mathcal{T}_{12}$, it follows from Lemma 2.3 and 2.4; we have

(2) is proved similarly.

$$
\begin{aligned}
d\left(A_{11} B_{12}\right)= & d\left(\frac{1}{2}\left(2 P \cdot A_{11} \cdot B_{12}+B_{12} \cdot A_{11} \cdot 2 P\right)\right) \\
= & \frac{1}{2}\left[d(2 P) A_{11} B_{12}+2 P d\left(A_{11}\right) B_{12}+2 A_{11} d\left(B_{12}\right)\right. \\
& \left.+2 d\left(B_{12}\right) A_{11}+2 B_{12} d\left(A_{11}\right) P+B_{12} A_{11} d(2 P)\right] \\
= & d\left(A_{11}\right) B_{12}+A_{11} d\left(B_{12}\right) .
\end{aligned}
$$

(3) For any $A_{11}, B_{11} \in \mathcal{T}_{11}, M_{12} \in \mathcal{T}_{12}$, by Lemma 2.5 (1), we get that

$$
d\left(A_{11} B_{11} M_{12}\right)=d\left(A_{11} B_{11}\right) M_{12}+A_{11} B_{11} d\left(M_{12}\right) .
$$

On the other hand,

$$
\begin{aligned}
d\left(A_{11} B_{11} M_{12}\right) & =d\left(A_{11}\left(B_{11} M_{12}\right)\right)=d\left(A_{11}\right) B_{11} M_{12}+A_{11} d\left(B_{11} M_{12}\right) \\
& =d\left(A_{11}\right) B_{11} M_{12}+A_{11} d\left(B_{11}\right) M_{12}+A_{11} B_{11} d\left(M_{12}\right) .
\end{aligned}
$$

This and Equation (1) imply that

$$
\left(d\left(A_{11} B_{11}\right)-d\left(A_{11}\right) B_{11}-A_{11} d\left(B_{11}\right)\right) M_{12}=0 .
$$

Since $P \mathcal{T Q}$ is a faithful left $P \mathcal{T P}$-module and $d\left(A_{11} B_{11}\right)-d\left(A_{11}\right) B_{11}-A_{11} d\left(B_{11}\right) \in \mathcal{T}_{11}$, we get $d\left(A_{11} B_{11}\right)-d\left(A_{11}\right) B_{11}-A_{11} d\left(B_{11}\right)=0$, that is

$$
d\left(A_{11} B_{11}\right)=d\left(A_{11}\right) B_{11}+A_{11} d\left(B_{11}\right) .
$$

Similarly, (4) is true for all $A_{22}, B_{22} \in \mathcal{T}_{22}$.

Lemma 2.6. $d\left(A_{11}+A_{12}\right)=d\left(A_{11}\right)+d\left(A_{12}\right)$ and $d\left(A_{12}+A_{22}\right)=d\left(A_{12}\right)+d\left(A_{22}\right)$.

Proof. Let $A_{11} \in \mathcal{T}_{11}, A_{12} \in \mathcal{T}_{12}$, it follows from Lemma 2.2 and 2.4, we have that $0=d\left(\frac{1}{2}\left(2 Q \cdot\left(A_{11}+A_{12}\right) \cdot Q+Q \cdot\left(A_{11}+A_{12}\right) \cdot 2 Q\right)\right)=Q d\left(A_{11}+A_{12}\right) Q$, that is, $d\left(A_{11}+A_{12}\right) \in \mathcal{T}_{11}+\mathcal{T}_{12}$.

For any $X_{12} \in \mathcal{T}_{12}$, it follows from Lemma 2.5 (1), we have

$$
d\left(A_{11} X_{12}\right)=d\left(A_{11}\right) X_{12}+A_{11} d\left(X_{12}\right) .
$$

On the other hand,

$$
\begin{aligned}
d\left(A_{11} X_{12}\right)= & d\left(\frac{1}{2}\left(2 P \cdot\left(A_{11}+A_{12}\right) \cdot X_{12}+X_{12} \cdot\left(A_{11}+A_{12}\right) \cdot 2 P\right)\right) \\
= & \frac{1}{2}\left[d(2 P)\left(A_{11}+A_{12}\right) X_{12}+2 P d\left(A_{11}+A_{12}\right) X_{12}+2\left(A_{11}+A_{12}\right) d\left(X_{12}\right)\right. \\
& \left.+2 d\left(X_{12}\right)\left(A_{11}+A_{12}\right) P+2 X_{12} d\left(A_{11}+A_{12}\right) P+X_{12}\left(A_{11}+A_{12}\right) d(2 P)\right] \\
= & d\left(A_{11}+A_{12}\right) X_{12}+A_{11} d\left(X_{12}\right) .
\end{aligned}
$$

This and Equation (2) imply that $\left[d\left(A_{11}+A_{12}\right)-d\left(A_{11}\right)\right] X_{12}=0$. Since $P \mathcal{T Q}$ is a faithful left $P \mathcal{T P}$-module; hence

$$
P\left[d\left(A_{11}+A_{12}\right)-d\left(A_{11}\right)\right] P=0 .
$$

Similarly, let $A_{12} \in \mathcal{T}_{12}$, for any $X_{22} \in \mathcal{T}_{22}$, then

$$
d\left(A_{12} X_{22}\right)=d\left(A_{12}\right) X_{22}+A_{12} d\left(X_{22}\right) .
$$

On the other hand,

$$
\begin{aligned}
d\left(A_{12} X_{22}\right) & =d\left(\frac{1}{2}\left(2 P \cdot\left(A_{11}+A_{12}\right) \cdot X_{22}+X_{22} \cdot\left(A_{11}+A_{12}\right) \cdot 2 P\right)\right) \\
& =d\left(A_{11}+A_{12}\right) X_{22}+A_{12} d\left(X_{22}\right) .
\end{aligned}
$$


Therefore, we get $\left[d\left(A_{11}+A_{12}\right)-d\left(A_{12}\right)\right] X_{22}=0$, that is

$P\left[d\left(A_{11}+A_{12}\right)-d\left(A_{12}\right)\right] Q+Q\left[d\left(A_{11}+A_{12}\right)-d\left(A_{12}\right)\right] Q=0$. So

$P\left[d\left(A_{11}+A_{12}\right)-d\left(A_{12}\right)\right] Q=0$ and $Q\left[d\left(A_{11}+A_{12}\right)-d\left(A_{12}\right)\right] Q=0$. Therefor combining Lemma 2.3, we have $d\left(A_{11}+A_{12}\right)-d\left(A_{11}\right)-d\left(A_{12}\right)=P\left[d\left(A_{11}+A_{12}\right)-d\left(A_{11}\right)-d\left(A_{12}\right)\right] P$ $+P\left[d\left(A_{11}+A_{12}\right)-d\left(A_{11}\right)-d\left(A_{12}\right)\right] Q+Q\left[d\left(A_{11}+A_{12}\right)-d\left(A_{11}\right)-d\left(A_{12}\right)\right] Q=0$. that is,

$d\left(A_{11}+A_{12}\right)=d\left(A_{11}\right)+d\left(A_{12}\right)$.

Similarly, (2) is true for all $A_{12} \in \mathcal{T}_{12}$ and $A_{22} \in \mathcal{T}_{22}$.

Lemma 2.7. $d\left(A_{11}+A_{12}+A_{22}\right)=d\left(A_{11}\right)+d\left(A_{12}\right)+d\left(A_{22}\right)$.

Proof. For any $A_{11} \in \mathcal{T}_{11}, A_{12} \in \mathcal{T}_{12}, A_{22} \in \mathcal{T}_{22}$,

$$
\begin{aligned}
& d\left(A_{11}\right)=d\left(\frac{1}{2}\left(P\left(A_{11}+A_{12}+A_{22}\right) P+P\left(A_{11}+A_{12}+A_{22}\right) P\right)\right)=P d\left(A_{11}+A_{12}+A_{22}\right) P . \\
& d\left(A_{12}\right)=d\left(\frac{1}{2}\left(2 P\left(A_{11}+A_{12}+A_{22}\right) Q+Q\left(A_{11}+A_{12}+A_{22}\right) 2 P\right)\right)=P d\left(A_{11}+A_{12}+A_{22}\right) Q . \\
& d\left(A_{22}\right)=d\left(\frac{1}{2}\left(Q\left(A_{11}+A_{12}+A_{22}\right) Q+Q\left(A_{11}+A_{12}+A_{22}\right) Q\right)\right)=Q d\left(A_{11}+A_{12}+A_{22}\right) Q .
\end{aligned}
$$

Thus,

$$
\begin{aligned}
d\left(A_{11}+A_{12}+A_{22}\right)= & \left.P d\left(A_{11}+A_{12}+A_{22}\right)\right) P+P d\left(A_{11}+A_{12}+A_{22}\right) Q \\
& +Q d\left(A_{11}+A_{12}+A_{22}\right) Q \\
= & d\left(A_{11}\right)+d\left(A_{12}\right)+d\left(A_{22}\right) .
\end{aligned}
$$

Lemma 2.8. For any $A_{12}, B_{12} \in \mathcal{T}_{12}$, we have $d\left(A_{12}+B_{12}\right)=d\left(A_{12}\right)+d\left(B_{12}\right)$.

Proof. For any $A_{12}, B_{12} \in \mathcal{T}_{12}$, from Lemma 2.3 and 2.6, we have

$$
\begin{aligned}
d\left(A_{12}+B_{12}\right)= & d\left(\frac{1}{2}\left(2 P \cdot\left(P+A_{12}\right) \cdot\left(Q+B_{12}\right)+\left(Q+B_{12}\right) \cdot\left(P+A_{12}\right) \cdot 2 P\right)\right) \\
= & P d\left(P+A_{12}\right)\left(Q+B_{12}\right)+P\left(P+A_{12}\right) d\left(Q+B_{12}\right) \\
& +d\left(Q+B_{12}\right)\left(P+A_{12}\right) P+\left(Q+B_{12}\right) d\left(P+A_{12}\right) P \\
= & P d\left(A_{12}\right) Q+P d\left(B_{12}\right)=d\left(A_{12}\right)+d\left(B_{12}\right) .
\end{aligned}
$$

Lemma 2.9. $d$ is additive on $\mathcal{T}_{11}$ and $\mathcal{T}_{22}$ respectively.

Proof. For any $A_{11}, B_{11} \in \mathcal{T}_{11}$, by Lemma 2.5 (1), we have

$$
d\left(\left(A_{11}+B_{11}\right) B_{12}\right)=d\left(A_{11}+B_{11}\right) B_{12}+\left(A_{11}+B_{11}\right) d\left(B_{12}\right) .
$$

on the other hand, from Lemma 2.5 (1) and 2.8, we get that

$$
d\left(\left(A_{11}+B_{11}\right) B_{12}\right)=d\left(A_{11} B_{12}+B_{11} B_{12}\right)=d\left(A_{11}\right) B_{12}+A_{11} d\left(B_{12}\right)+d\left(B_{11}\right) B_{12}+B_{11} d\left(B_{12}\right) .
$$

This and Equation (3) imply that

$$
\left(d\left(A_{11}+B_{11}\right)-d\left(A_{11}\right)-d\left(B_{11}\right)\right) B_{12}=0 .
$$

Since $P \mathcal{T Q}$ is a faithful left $P \mathcal{T P}$-module and $d\left(A_{11}+B_{11}\right)-d\left(A_{11}\right)-d\left(B_{11}\right) \in \mathcal{T}_{11}$, we have that $d\left(A_{11}+B_{11}\right)-d\left(A_{11}\right)-d\left(B_{11}\right)=0$, that is

$$
d\left(A_{11}+B_{11}\right)=d\left(A_{11}\right)+d\left(B_{11}\right) .
$$

Similarly, we can also get the additivity of $d$ on $\mathcal{T}_{22}$.

Lemma 2.10. $d$ is additivity.

Proof. For any $A, B \in \mathcal{T}$, write $A=A_{11}+A_{12}+A_{22}, B=B_{11}+B_{12}+B_{22}$, where $A_{i j}, B_{i j} \in \mathcal{T}_{i j}, 1 \leq i \leq j \leq 2$. Then Lemma 2.7-2.9 are all used in seeing the equation 


$$
\begin{aligned}
d(A+B) & =d\left(A_{11}+A_{12}+A_{22}+B_{11}+B_{12}+B_{22}\right) \\
& =d\left(A_{11}+B_{11}\right)+d\left(A_{12}+B_{12}\right)+d\left(A_{22}+B_{22}\right) \\
& =d\left(A_{11}\right)+d\left(B_{11}\right)+d\left(A_{12}\right)+d\left(B_{12}\right)+d\left(A_{22}\right)+d\left(B_{22}\right) \\
& =d\left(A_{11}+A_{12}+A_{22}\right)+d\left(B_{11}+B_{12}+B_{22}\right) \\
& =d(A)+d(B) .
\end{aligned}
$$

Lemma 2.11. $d(A B)=d(A) B+A d(B)$ for all $A, B \in \mathcal{T}$.

Proof. For any $A, B \in \mathcal{T}$, let $A=A_{11}+A_{12}+A_{22}, B=B_{11}+B_{12}+B_{22}$, where $A_{i j}, B_{i j} \in \mathcal{T}_{i j}, 1 \leq i \leq j \leq 2$. Now we have that by Lemma 2.5 (1)-(4), Lemma 2.7 and 2.8

$$
\begin{aligned}
d(A B)= & d\left(A_{11} B_{11}+A_{11} B_{12}+A_{12} B_{22}+A_{22} B_{22}\right) \\
= & d\left(A_{11} B_{11}\right)+d\left(A_{11} B_{12}\right)+d\left(A_{12} B_{22}\right)+d\left(A_{22} B_{22}\right) \\
= & d\left(A_{11}\right) B_{11}+A_{11} d\left(B_{11}\right)+d\left(A_{11}\right) B_{12}+A_{11} d\left(B_{12}\right) \\
& +d\left(A_{12}\right) B_{22}+A_{12} d\left(B_{22}\right)+d\left(A_{22}\right) B_{22}+A_{22} d\left(B_{22}\right) .
\end{aligned}
$$

On the other hand, it follows from Lemma 2.3, 2.7; we get that

$$
\begin{aligned}
& d(A) B+A d(B) \\
= & d\left(A_{11}+A_{12}+A_{22}\right)\left(B_{11}+B_{12}+B_{22}\right)+\left(A_{11}+A_{12}+A_{22}\right) d\left(B_{11}+B_{12}+B_{22}\right) \\
= & d\left(A_{11}\right) B_{11}+A_{11} d\left(B_{11}\right)+d\left(A_{11}\right) B_{12}+A_{11} d\left(B_{12}\right) \\
& +d\left(A_{12}\right) B_{22}+A_{12} d\left(B_{22}\right)+d\left(A_{22}\right) B_{22}+A_{22} d\left(B_{22}\right) .
\end{aligned}
$$

It is clear that $d(A B)=d(A) B+A d(B)$ for all $A, B \in \mathcal{T}$.

Proof of Theorem 2.1. From the above lemmas, we have proved that $d$ is an additive derivation on $\mathcal{T}$. Since $d(A)=\delta(A)-(A T-T A)$ for each $A \in \mathcal{T}$, by a simple calculation, we see that $\delta$ is also an additive derivation. The proof is completed.

\section{Acknowledgements}

The author would like to thank the editors and the referees for their valuable advice and kind helps.

\section{References}

[1] Brešar, M. (1989) Jordan Mappings of Semiprime Rings. Journal of Algebra, 127, 218-228. http://dx.doi.org/10.1016/0021-8693(89)90285-8

[2] Herstein, I.N. (1969) Topics in Ring Theory. University of Chicago Press, Chicago, London.

[3] Wei, F. and Xiao, Z.K. (2009) Generalized Jordan Triple Higher Derivations on Semiprime Rings. Bulletin of the Korean Mathematical Society, 46, 553-565. http://dx.doi.org/10.4134/BKMS.2009.46.3.553

[4] Li, J.K. and Lu, F.Y. (2007) Additive Jordan Derivations of Reflexive Algebras. Journal of Mathematical Analysis and Applications, 329, 102-111. http://dx.doi.org/10.1016/j.jmaa.2006.06.019

[5] Zhang, J.H. (1998) Jordan Derivations on Nest Algebras. Acta Mathematica Sinica, Chinese Series, 41, 205-213. (In Chinese)

[6] Fošner, M. and Ilišević, D. (2008) On Jordan Triple Derivations and Related Mappings. Mediterranean Journal of Mathematics, 5, 1660-5454. http://dx.doi.org/10.1007/s00009-008-0159-9

[7] Jing, W. and Lu, S. (2003) Generalized Jordan Derivations on Prime Rings and Standard Operator Algebras. Taiwanese Journal of Mathematics, 7, 605-613.

[8] Shang, Y. (2013) On the Ideals of Commutative Local Rings. Kochi Journal of Mathematics, 8, 13-17.

[9] Shang, Y. (2011) A Study of Derivations in Prime Near-Rings. Mathematica Balkanica (N.S.), 25, 413-418.

[10] Cheung, W.S. (2000) Mappings on Triangular Algebras. Ph.D. Dissertation, University of Victoria, British Columbia, Canada.

[11] Zhang, J.H. and Yu, W.Y. (2006) Jordan Derivations of Triangular Algebras. Linear Algebra and Its Applications, 419, 251-255. http://dx.doi.org/10.1016/j.laa.2006.04.015 
Scientific Research Publishing (SCIRP) is one of the largest Open Access journal publishers. It is currently publishing more than 200 open access, online, peer-reviewed journals covering a wide range of academic disciplines. SCIRP serves the worldwide academic communities and contributes to the progress and application of science with its publication.

Other selected journals from SCIRP are listed as below. Submit your manuscript to us via either submit@scirp.org or Online Submission Portal.
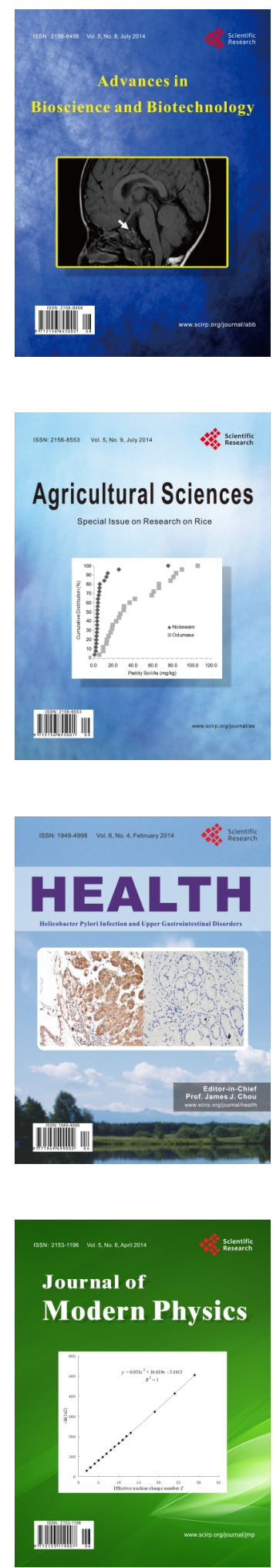
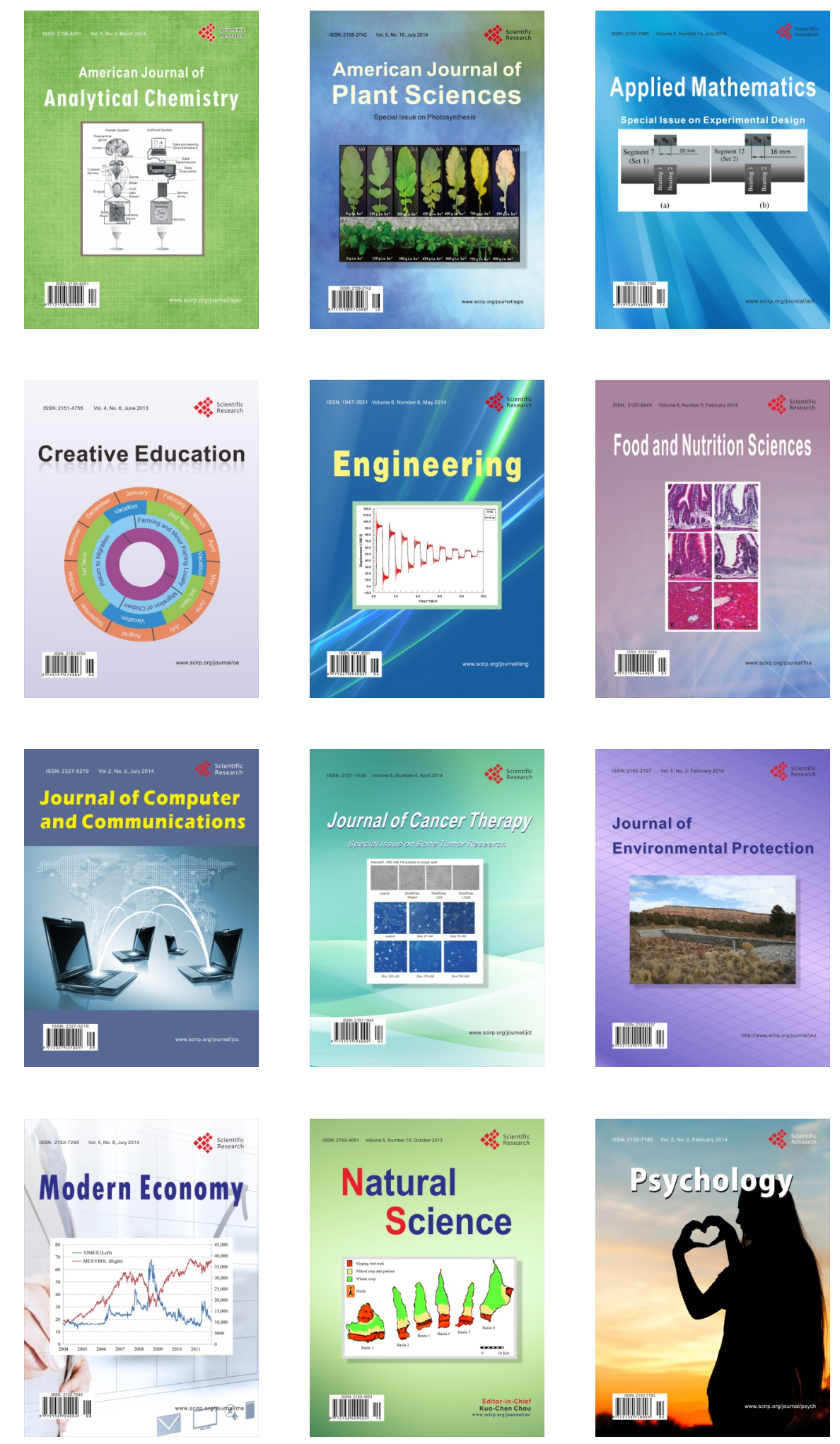